Revue internationale P.M.E.

Économie et gestion de la petite et moyenne entreprise

\title{
Évaluation du risque lié à une innovation pour les PME: proposition d'un outil
}

\section{Sophie Reboud et Tim W. Mazzarol}

Volume 19, numéro 2, 2006

URI : https://id.erudit.org/iderudit/1008498ar

DOI : https://doi.org/10.7202/1008498ar

Aller au sommaire du numéro

\section{Éditeur(s)}

Presses de l’Université du Québec

ISSN

0776-5436 (imprimé)

1918-9699 (numérique)

Découvrir la revue

Citer cet article

Reboud, S. \& Mazzarol, T. W. (2006). Évaluation du risque lié à une innovation pour les PME: proposition d'un outil. Revue internationale P.M.E., 19(2),

133-161. https://doi.org/10.7202/1008498ar

\section{Résumé de l'article}

Les petites et moyennes entreprises (PME) sont souvent caractérisées par un taux élevé d'innovation et un engagement marqué dans une démarche d'innovation comme moyen de construire un avantage concurrentiel. Il reste cependant difficile pour les managers de telles entreprises d'évaluer correctement les mérites d'une innovation donnée, en particulier en termes de possibilité de retour sur investissement. Pour répondre à ce besoin, nous avons élaboré en 2002-2003, pour l'INPI, un outil d'analyse pour aider les entrepreneurs à évaluer le profil de risque attaché à l'investissement dans le développement d'une innovation. La première version de ce modèle propose une mesure de la «rente», ou retour financier, attendue d'un investissement dans une innovation donnée. Durant l'été 2003, nous avons poursuivi le développement de cet outil avec une enquête pilote sur un échantillon de petites firmes innovantes d'Australie occidentale. Le questionnaire conçu a été complété en ajoutant des éléments permettant de déterminer les influences relatives des "Attitudes et Normes subjectives» sur le processus de décision de l'entrepreneur. Cette étude préliminaire, dont les résultats sont en cours d'analyse, a pour objectif d'apporter des éléments d'évaluation des pratiques d'innovation des PME.
Ce document est protégé par la loi sur le droit d'auteur. L'utilisation des services d'Érudit (y compris la reproduction) est assujettie à sa politique d'utilisation que vous pouvez consulter en ligne.

https://apropos.erudit.org/fr/usagers/politique-dutilisation/ 


\title{
Évaluation du risque lié à une innovation pour les PME: proposition d'un outil'
}

\author{
Sophie REBOUD \\ CEREN, Groupe ESC Dijon-Bourgogne
}

Tim W. MAZZAROL

CEMI, Graduate School of Management

University of Western Australia

MOTS CLÉS

PME - Innovation - Processus de décision - Évaluation du risque Management stratégique - Entrepreneur

\begin{abstract}
LES AUTEURS
SopHIE ReBoud est directrice du Centre de recherche sur les entreprises (CEREN) et professeure de stratégie et innovation au Groupe ESC Dijon-Bourgogne. Elle possède 10 ans d'expérience comme chercheure et consultante dans les domaines du management et de la stratégie. Elle est titulaire d'un diplôme d'ingénieur agronome de l'INA-PG et d'un doctorat en économie industrielle de l'École des mines de Paris. Adresse: Groupe ESC DijonBourgogne, 29, rue Sambin, B.P. 50608, 21006 Dijon. Téléphone: 03807259 00, télécopieur: 03807259 99. Courriel: <sreboud@escdijon.com>. Site Web: <http://www.escdijon.com>.

TIM W. MAZZAROL est directeur du Center for Entrepreneurial Management and Innovation (CEMI) et professeur associé en entrepreneuriat et stratégie à la Graduate School of Management de I'Université Western Australia. II possède 15 ans d'expérience professionnelle, aussi bien dans des petites firmes entrepreneuriales que dans des grandes entreprises ou administrations. Ses recherches sur les PME ont été publiées internationalement. II est titulaire d'un Ph.D. en management et d'un MBA avec mention de l'Université de Curtin, en Australie occidentale.
\end{abstract}

1. Une version préliminaire de ce travail est parue en anglais en octobre 2003 dans les Cahiers de recherche du CEREN et une version intermédiaire de ce travail a été présentée en français à la XXIII ${ }^{e}$ Conférence de l'AIMS.

Tous nos remerciements vont aux trois évaluateurs anonymes pour leurs judicieux conseils et commentaires. Nous restons néanmoins responsables des manques et imperfections qui subsisteraient dans cette version. 


\begin{abstract}
RÉSUMÉ
Les petites et moyennes entreprises (PME) sont souvent caractérisées par un taux élevé d'innovation et un engagement marqué dans une démarche d'innovation comme moyen de construire un avantage concurrentiel. II reste cependant difficile pour les managers de telles entreprises d'évaluer correctement les mérites d'une innovation donnée, en particulier en termes de possibilité de retour sur investissement. Pour répondre à ce besoin, nous avons élaboré en 2002-2003, pour l'INPI, un outil d'analyse pour aider les entrepreneurs à évaluer le profil de risque attaché à l'investissement dans le développement d'une innovation. La première version de ce modèle propose une mesure de la «rente», ou retour financier, attendue d'un investissement dans une innovation donnée. Durant l'été 2003, nous avons poursuivi le développement de cet outil avec une enquête pilote sur un échantillon de petites firmes innovantes d'Australie occidentale. Le questionnaire conçu a été complété en ajoutant des éléments permettant de déterminer les influences relatives des "Attitudes et Normes subjectives" sur le processus de décision de l'entrepreneur. Cette étude préliminaire, dont les résultats sont en cours d'analyse, a pour objectif d'apporter des éléments d'évaluation des pratiques d'innovation des PME.
\end{abstract}

\begin{abstract}
Small and medium enterprises (SMEs) are frequently associated with high levels of innovation and a commitment to innovative behaviour as a means of achieving competitive advantage. However, it is difficult for the entrepreneurial managers of such SMEs to fully assess the merits of a particular innovation, particularly in relation to its risk-return trade off. To address this need, we have commenced in 2002, in conjunction with INPI, the development of a screening tool designed to assist entrepreneurs evaluate the risk-return profile of future innovation investment. The preliminary model developed measures the anticipated "rent" or financial return expected from a particular investment in an innovation. During 2003, we further developped the tool with a pilot survey of small innovative firms in Western Australia. An additional component of the study sought to determine the relative influences of Attitude and Subjective Norm on the decision-making process of the entrepreneur. The aim of the study is to propose more evaluation criteria for the management process of innovation.
\end{abstract}

\title{
RESUMEN
}

Las Pequeñas y Medianas Empresas (PyMEs) se caracterizan a menudo por una elevada tasa de innovación y un compromiso inscrito dentro del proceso de innovación como medio para construir una ventaja competitiva. Sigue siendo sin embargo difícil para los «managers» de tales empresas evaluar correctamente los méritos de una innovación dada, particularmente en términos de posibilidad de retorno sobre la inversión. Para responder a esta necesidad, hemos elaboradó en 2002-2003 para el INPI una herramienta de análisis para ayudar a los empresarios a evaluar el perfil de riesgo vinculado a la inversión en el desarrollo de una innovación. La primera versión de este modelo propone una medición de la «renta», o retorno financiero esperado de una inversión en una innovación dada. Durante el verano 2003, hemos continuado el desarrollo de esta herramienta con una encuesta piloto sobre una 
muestra de pequeñas firmas innovadoras de Australia Occidental. El cuestionario concebido ha sido completado agregando elementos que permitan determinar las influencias relativas de las "Actitudes y Normas Subjetivas» sobre el proceso de decisión del empresario.

\section{ZUSAMMENFASSUNG}

Kleine und mittlere Unternehmen (KMU) werden oftmals durch einen hohen Innovationsgrad und einem starken Engagement zur eigenen Innovationsförderung (Bildung von Wettbewerbsvorteilen) charakterisiert. Für Unternehmensführer solcher Betriebe bleibt es jedoch schwierig die Resultate von Innovationen abzuschätzen, im Besonderen gilt dies für den ROI. Um dieses Bedürfnis abzudecken, haben wir in den Jahren 2002 und 2003 am Institut „INPI" ein Analyseinstrument entwickelt, dass es erlaubt, ein Risikoprofil für Investitionen in Innovationsprojekte zu erstellen. Die erste Version dieses Modells behilft sich einer Messung der Rente (finanzielle Mehrerträge), die von einer Investition in eine bestimmte Innovation erwartet wird. Während dem Sommer 2003 haben wir das Messinstrument mit der Hilfe einer Pilotstudie bei einer Auswahl von innovativen Firmen in Westaustralien weiter verfeinert. Dabei wurde unser Fragebogen mit Aussagen vervollständigt, die den Einfluss von subjektiven Einstellungen und Normen des Unternehmers auf seine Entscheide dokumentieren. Diese einleitende Studie, deren Resultate aktuell analysiert werden, hat zum Ziel, Bewertungsmassstäbe zu definieren, die die Innovationspraktiken der KMU umschreiben vermögen.

\section{Introduction}

Cet article a pour objectif de poser les bases d'une recherche de long terme comprenant plusieurs étapes. Nous établirons dans une première partie les origines de la recherche ainsi que le cadre conceptuel choisi. Ensuite, nous présenterons l'outil d'analyse et de conseil qui est issu de la première phase de la recherche. Enfin, nous proposerons un ensemble de questions de recherche qui seront traitées dans une phase ultérieure de l'étude.

\section{Origine de la recherche}

Le point de départ de cette recherche est une demande de l'INPI (Institut national de la propriété industrielle) à une équipe de chercheurs en management stratégique de concevoir un outil d'analyse et de conseil pour aider les innovateurs et les managers de petites entreprises à évaluer rapidement le risque stratégique que pose la mise en œuvre d'un projet d'innovation. Plus précisément, l'INPI souhaitait, grâce à cet outil, d'une part, former ses propres conseillers à la place de la propriété industrielle dans la stratégie et, d'autre part, guider le dialogue qui s'établit entre un conseiller en propriété industrielle et un porteur d'innovation cherchant à se protéger 
avant de se lancer sur le marché. Cet outil était donc destiné à aider un innovateur ou son conseiller à choisir ex ante une stratégie adaptée aux caractéristiques de l'innovation: la lancer sur le marché ou non, la protéger ou non, envisager de nouer un partenariat avec un fournisseur, un client ou un concurrent.

Pour réaliser ce travail, nous avons construit un cadre conceptuel dans lequel nous avons modélisé la réflexion que pourrait avoir un innovateur cherchant à envisager la valeur qu'il pourra retirer de son innovation. Nous allons donc d'abord présenter les fondements du cadre conceptuel et son contexte avant de détailler les différentes étapes de la modélisation.

\subsection{Innovation, risque et PME, nécessité d'un outil d'évaluation}

Les petites entreprises (moins de 250 salariés) ne représentent pas seulement la forme d'entreprise la plus courante dans la plupart des économies, elles sont également une source très importante d'innovations (Freel, 2000; Hansen, Sondergard et Meredith, 2002; Mogee, 2000). Par exemple, aux États-Unis, les petites firmes entrepreneuriales ont été créditées de $67 \%$ des inventions et de $95 \%$ des innovations radicales depuis 1945 (NCOE, 2000). De plus, ce phénomène a été observé dans de nombreuses autres économies, par exemple, dans l'Union européenne (European Commission, 2004) et en Asie (APEC, 2003). En 2004, en Europe (Union à 25), plus de la moitié des PME ont déclaré avoir réalisé une innovation de procédés (54\% des moins de 50 salariés, $50 \%$ des moins de 250), plus encore une innovation de produit ou service ( $73 \%$ des moins de $50 ; 76 \%$ des moins de 250 salariés).

L'innovation en contexte concurrentiel implique une activité entrepreneuriale, au sens schumpétérien du terme, généralement sous la forme de nouveaux produits, de nouveaux procédés de production et de nouvelles activités ayant la capacité de créer un potentiel de croissance économique ou sociale (Drucker, 2002; Huet, 2004). Par sa nature, l'innovation implique un changement qui impose à son tour une prise de risque à l'entreprise cherchant à la développer. Ce risque, lié à l'incertitude technique (Ravichandran, 2001), commerciale et financière quant aux réelles possibilités de l'innovation, est inhérent au phénomène d'innovation lui-même (Gatignon et Robertson, 1993; Dziura, 2001; Tornatzky et Fleischer, 1990 ) et vient s'ajouter aux risques habituellement pris en compte dans les modèles d'analyse financière des investissements ${ }^{2}$. On peut, avec Klinke et Renn (2002), définir ce risque comme «la possibilité que les actions humaines ou des événements affectent ce que les humains valorisent». Ici, c'est en effet l'incertitude globale

2. Pour simplifier, on pourra distinguer le risque opérationnel (portant sur la rentabilité brute de l'investissement), le risque systématique (lié au secteur d'activité dans lequel s'effectue l'investissement) et le risque de taux (se rapportant à l'estimation du taux d'actualisation utilisé pour évaluer la VAN). 
liée à l'innovation qui conduit les adopteurs potentiels de l'innovation à se réfugier dans une attitude attentiste et à augmenter aussi les efforts à développer pour les convaincre (Arthur, 1996; Shapiro et Varian, 1998). L'évaluation du risque liée à une innovation donnée est ainsi une étape critique de l'évaluation de l'innovation elle-même (Guilhon et Montchaud, 2003).

\subsection{Une différence fréquente entre la valeur espérée et la valeur effectivement captée}

Les modèles financiers (par exemple, le «payback», souvent utilisé par les PME, ou la VAN [valeur actuelle nette] ou encore le TRI [taux de rendement interne]) peuvent donner des indications quant à l'attractivité potentielle des innovations, néanmoins, d'une part, ces mesures restent limitées dans leur capacité à évaluer correctement les aspects non financiers pouvant avoir un impact sur l'investissement, en particulier les réactions concurrentielles des acteurs de l'environnement ${ }^{3}$, d'autre part, elles supposent une estimation fine des flux financiers attendus, ce qui se révèle difficile ex ante. De plus, les innovateurs sont souvent convaincus de la valeur de leur innovation mais peuvent éprouver des difficultés à l'analyser, voire à en démontrer la réalité. Trois raisons principales peuvent expliquer cela.

- Premièrement, l'innovateur attache souvent beaucoup d'importance à la valeur absolue anticipée, sans prendre en considération les difficultés pouvant survenir lors du lancement de l'innovation (Martin et Scott, 2000). Cette évaluation risque alors d'être excessivement optimiste, soit parce que la valeur de l'innovation elle-même a été surestimée, soit parce qu'auront été sous-estimés les prélèvements effectués, grâce à leurs pouvoirs de négociation, par les acteurs de l'environnement comme les fournisseurs, les clients ou les concurrents.

- Deuxièmement, les innovateurs suivent fréquemment un mode de raisonnement peu structuré, basé plus sur des intuitions que sur des raisonnements rationnels (Mockler, 2003). De plus, les décideurs ont tendance à considérer les problèmes comme uniques et donc à déconnecter leur décision d'expériences passées ou de conséquences futures probables (Kahneman et Lovallo, 1993). La probabilité est d'autant plus forte si l'innovateur «dirigeant-propriétaire» est relativement isolé et manque du soutien d'une équipe compétente pour l'aider à mener correctement une étude de faisabilité.

3. Voir la recension effectuée par Beaudoin et St-Pierre (1999) pour plus de détails. Ces modèles pourront analyser certains aspects du contexte dans lequel l'innovation s'insérera, mais ne donneront pas d'indication sur les modifications que l'innovation ne manquera pas de susciter, en particulier en termes de réaction des acteurs. 
- Troisièmement, l'innovation requiert fréquemment un processus complexe, non linéaire, caractérisé par de nombreuses boucles de rétroaction, ce qui renforce la difficulté d'estimation de sa valeur ex ante. Ainsi que le note Jensen (1982) «[...] when the innovation is introduced, the firm does not know whether adoption will be profitable or not, but this uncertainty can be reduced by waiting and gathering information».

Au total, on peut souhaiter un outil d'évaluation du risque, permettant à des dirigeants de PME d'évaluer ex ante et de façon approchée, correspondant à la réflexion et à la recherche d'information mentionnée par Jensen (1982), le risque que leur innovation ne leur rapporte pas autant d'argent que ce qu'ils pouvaient espérer. Comme précisé plus haut, l'idée sous-tendant la conception de cet outil est qu' «il vaut mieux avoir raison de façon large que tort de façon précise» et que, pour prendre une décision de poursuivre les essais et le lancement d'une innovation, un patron de PME cherche plus à conforter son intuition qu'à calculer les retours précis qu'il peut en attendre. Ce point de départ fort peu scientifique permet néanmoins d'étudier ensuite la pertinence des choix faits en matière d'innovation et de les corréler avec un mode de management de l'innovation.

Autrement dit, cet outil doit permettre d'évaluer le montant d'argent que l'innovateur pourrait espérer toucher si tout se passait sans difficulté et l'amener, étape par étape, à estimer tout ce que les éventuelles réactions de l'environnement pourraient lui coûter. Par ailleurs, l'appui d'un tel outil peut aider les PME à convaincre, grâce à des arguments plus solides, des financeurs d'innovation de les suivre dans leur projet (ce qui sinon se révèle quasi impossible [voir Guilhon et Montchaud, 2003, ou Bach et Lambert, 2000]).

Dans cet article, nous présentons tout d'abord l'outil lui-même et sa logique de construction, puis les analyses passées, actuelles et futures, entreprises pour mesurer l'intérêt de cet outil et de son utilisation potentielle par les dirigeants de PME.

\section{L'outil d'analyse développé pour l'INPI}

\subsection{Objectifs et logique du modèle: des recommandations sur le choix d'une stratégie de valorisation des innovations}

L'innovation dans la théorie économique est une source potentielle d'avantage concurrentiel pour les entreprises (Nemeth, 1997). La rente qui lui est associée est la condition pour le succès futur de son développement. Ainsi, un entrepreneur innovateur va développer une nouvelle ressource ou compétence ou va créer une nouvelle combinaison de ressources et compétences existantes (Schumpeter, 1912). Une entreprise ayant acquis un avantage concurrentiel pourra obtenir un taux de 
profit supérieur au taux de profit tiré en moyenne dans son activité et surtout sera à même de maintenir ce taux supérieur pendant une certaine période. Dans de nombreux cas d'innovation ${ }^{4}$, cette durabilité de l'avantage concurrentiel peut être protégée grâce au système des brevets ${ }^{5}$. L'innovateur est alors le seul utilisateur légal de l'innovation pendant une période de 20 ans, ce qui lui garantit un monopole légal et crée donc un avantage concurrentiel soutenable, auquel on peut rattacher le concept de rente ricardienne. Par extension, nous utiliserons également le terme de rente lorsque le mode de protection et de valorisation de l'innovation ne passe pas par un brevet. L'objectif du modèle est justement d'amener l'innovateur à choisir le mode de valorisation le plus adapté à son innovation et à sa situation concurrentielle.

\subsubsection{Le modèle}

Le modèle tel qu'il a été conçu permet d'estimer la «rente» (au sens ricardien ${ }^{6} \mathrm{du}$ terme) anticipée lors de l'investissement dans une innovation donnée (Miles, Paul et Wilhite, 2003). Pour aider l'innovateur à aller plus loin que la simple estimation de la valeur initialement attribuée à l'innovation, mais aussi les effets d'érosion auxquels elle sera confrontée lors de son introduction sur un marché, le processus d'analyse est constitué de plusieurs étapes ${ }^{7}$ (Santi et al., 2003; $c f$. figure 1).

1. Analyse de la rente initialement anticipée ou «rente potentielle», en relation avec le type d'innovation et la taille des marchés clients potentiels.

4. Nombreux en général, mais peu répandus chez les PME. L'innobaromètre européen 2004 relève par exemple que les PME présentent «une insuffisante prise en compte de la propriété intellectuelle», seules $12 \%$ ont déclaré avoir protégé leurs innovations par des brevets.

5. Une revue très complète sur ce thème peut être trouvée dans le numéro spécial de la Revue d'économie industrielle, $\mathrm{n}^{\circ} 99,2^{\mathrm{e}}$ trimestre 2002. Nous verrons néanmoins que la volonté de protection de la rente à venir peut n'être qu'un des objectifs de la demande de brevet (voir à ce sujet Corbel, 2003).

6. Pour une analyse approfondie du concept de rente, voir, par exemple, Schumpeter (1912), Lewin et Phelan (2002) et Dagnino (1996). Empruntée à l'économie néoclassique, elle représente un surplus, un excès de gain par rapport à une situation considérée comme normale. On en distingue quatre principales sortes, dont la rente ricardienne provenant d'une ressource dont on est propriétaire (terrain, brevet). Les autres sont la rente de monopole, la rente entrepreneuriale et la quasi-rente. Dans un premier temps, nous cherchons à évaluer le potentiel de rente qu'un innovateur peut espérer obtenir de son innovation. L'analyse du type de rente peut ensuite permettre de déterminer le choix stratégique à faire pour protéger l'innovation contre les forces concurrentielles probables. Notons que dans l'utilisation de l'outil pour guider les PME, les conseillers ou les consultants disposent d'un glossaire pour les aider, le cas échéant, à préciser et expliquer les termes techniques.

7. Qui sont bien des étapes de l'analyse et non pas une progression au cours du temps.

Revue internationale P.M.E., vol. 19, nº 2, 2006 
2. Analyse des caractéristiques de l'environnement des marchés potentiels, et des effets d'érosion liés à ces caractéristiques puis analyse des pressions concurrentielles auxquelles l'innovation sera confrontée et de la rente «résiduelle» qui résultera de l'érosion de la rente potentielle après effet de ces pressions.

3. Analyse de la situation compétitive de la PME et de sa capacité à lancer l'innovation et à capter tout ou partie de la rente qu'on appellera ainsi rente «appropriable».

Figure 1

\section{Logique du processus d'analyse}

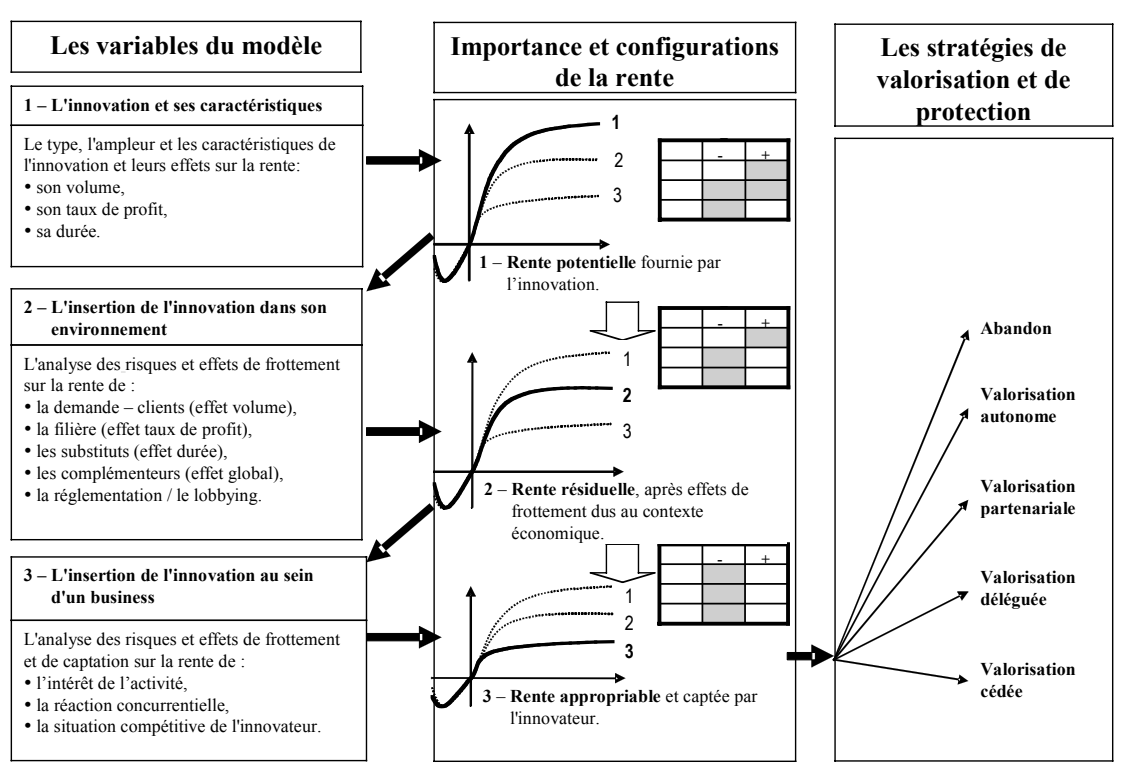

Source: Santi et al., 2003.

\subsection{Le modèle d'évaluation du risque}

\subsubsection{La rente potentielle}

Pour analyser l'intérêt ex ante d'une innovation, il est nécessaire de mesurer le montant total de rente qu'elle est susceptible de fournir. Ce montant peut être approché par la combinaison de trois composantes qui peuvent varier indépendamment les unes des autres ( $c f$. figure 2). 
1. Le volume - mesuré par le chiffre d'affaires annuel anticipé réalisé grâce à l'innovation et estimé grâce à trois indicateurs accessibles évaluant la taille du marché client ciblé, le nombre de pays éventuellement concernés par l'innovation, le nombre d'applications possibles de l'innovation au-delà de son utilisation d'origine. Notons que cette première estimation du volume potentiel se fait sur la base de la taille du marché cible, indépendamment de l'envie que les clients ont d'acheter l'innovation. Nous avons choisi d'analyser ce critère plus loin, comme un facteur d'érosion de la rente potentielle. La raison principale est qu'il peut être possible de «retravailler» l'innovation pour mieux tenir compte des envies des clients ciblés.

2. Le taux de marge - mesuré par la marge anticipée réalisée grâce à l'innovation, ramenée au CA et estimé grâce à deux indicateurs principaux: l'un lié à la nature de l'innovation (plus elle est incrémentale, plus les marges espérées sont faibles) et son mode de génération en fonction du nombre d'acteurs concernés (Pavitt, 1984) ${ }^{8}$.

3. La durée - mesurée par la durée d'exploitation probable de l'innovation, qui variera en fonction de l'origine de l'innovation (ou ses bases technologiques: plus on est en amont, vers la science fondamentale, plus la durée anticipée peut être longue ${ }^{9}$ ), en fonction de la réceptivité à l'adoption des adopteurs ciblés et de la difficulté à copier techniquement l'innovation.

Ces caractéristiques de la rente issue de l'innovation peuvent varier de façon indépendante: un nouveau jeu vidéo, par exemple, pourra engendrer un fort volume, mais sa durée sera faible; en revanche, la photographie Polaroïd aura généré un faible volume et sa durée de vie aura été longue. Autre exemple, la poêle Tefal sera caractérisée par un volume fort, une durée longue mais un faible taux; au contraire, avec un gadget comme le Hulla hoop, on aura un faible volume et une faible durée mais un taux important. L'intérêt principal de l'analyse séparée de ces composantes est qu'elle permet de distinguer les facteurs supposant une vigilance particulière lors de la confrontation de l'innovation avec les forces concurrentielles:

8. Mode de génération de l'innovation: cet indicateur reprend en partie et en l'aménageant la taxonomie de Pavitt sur les trajectoires technologiques. Il la modifie et l'améliore en distinguant, en particulier, plusieurs variantes dans le cas «système» jugées d'effet d'importance croissante sur le taux potentiel de la rente.

9. Fondements technologiques de l'innovation: cet indicateur lie le caractère durable de l'innovation aux bases technologiques qui la fondent - fondamentales s'appuyant un «long» temps et travail de recherche (hors et dans l'entreprise innovatrice) vs appliquées et avec un temps de recherche relativement court. 
si, par exemple, l'intérêt principal d'une innovation est son volume, on surveillera particulièrement les facteurs sources d'un volume important (utilité perçue par le client, par exemple).

FIGURE 2

\section{Composantes de la rente}

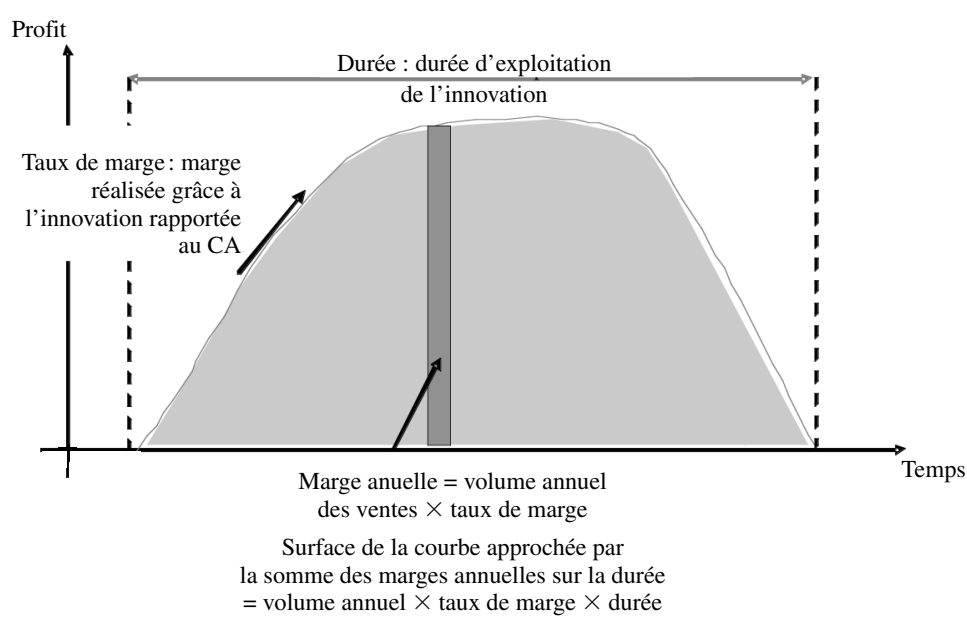

Source: Santi et al., 2003.

Ainsi nous obtenons: $\quad$ rente $=$ volume $\times$ taux $\times$ durée.

Avant d'introduire l'innovation sur le marché, la première étape pour la PME devrait donc consister à estimer le montant prévisible de la rente potentielle. Pour cela, elle devrait donc mesurer le volume, le taux et la durée de la rente anticipée susceptible d'être engendrée par l'innovation avant interaction avec les forces du marché. Les indicateurs proposés pour mener cette analyse sont présentés dans le tableau 1.

Deux caractéristiques vont prendre une importance toute particulière dans l'évaluation de l'innovation et peuvent avoir une forte influence sur la suite de l'analyse.

1. Le caractère systémique versus isolé de l'innovation (Henderson et Clark, 1990) ${ }^{10}$. C'est l'un des critères de la typologie des innovations utilisée

10. L'innovation isolée désigne une innovation technologique qui peut fonctionner de façon autonome et isolée (par exemple, un oreiller antibactérien est une innovation isolée), par opposition, l'innovation systémique désigne une innovation technologique qui ne peut fonctionner seule et qui, pour réussir, nécessite des compléments ou l'insertion dans un 
dans ce travail, avec des effets sur les recommandations en matière de protection. Les réactions des acteurs à anticiper seront plus nombreuses et complexes dans le cas d'une innovation systémique (par exemple, l'influence des acteurs complémenteurs).

TABLEAU 1

Mesure de la rente potentielle

\section{Volume d'affaires potentiel}

Le volume d'affaires potentiel correspond au chiffre d'affaires annuel moyen potentiellement réalisable sur l'ensemble des marchés d'application possibles (une somme de CA annuel).

\section{Taux de marge potentiel}

Le taux de marge potentiel est totalement lié à la valeur et l'importance de l'avantage concurrentiel créé par l'innovation.

\section{Indicateurs pertinents}

- Potentiel de diffusion sectoriel de l'innovation. Le spectre des applications potentiel est: de très limité (1 niche) à très large (multitude d'applications).

- Potentiel de diffusion géographique de l'innovation. Le marché naturel est: de purement français à mondial.

- Taille des marchés clients: de moins de $50 \mathrm{M€}$ à plus de $3 G €$.

- Limite d'exploitation. Les revendications de brevets antérieurs interdisent: de toutes les applications à aucune application.
Indicateurs pertinents

- Mode de génération de l'innovation:

depuis en isolation $d u$ marché jusqu'à avec un réseau large et diversifié.

- Type d'innovation: de la plus incrémentale à la plus radicale.

- Nature de la protection antérieure:

depuis une invention déjà divulguée et publique à une innovation libre de toute protection antérieure.

\section{Durée potentielle} d'exploitation de la rente

La durée d'exploitation potentielle de la rente, indépendamment de son mode de protection, est liée au caractère durable (et défendable) de l'innovation, en soi et relativement au secteur où elle est commercialisée.

\section{Indicateurs pertinents}

- Fondements technologiques de l'innovation:

depuis un procédé simple jusqu'à un nouveau paradigme scientifique.

- Intensité «innovative» du secteur d'application, c'està-dire le taux de renouvellement des innovations et produits:

de très forte (défavorable) à très faible (favorable).

- Copiabilité de l'innovation: technique (les caractéristiques de l'innovation la rendent très facilement, à très difficilement, copiable), juridique (en fonction de la protection, elle est très facile à très difficile à copier).

Source: Santi et al., 2003.

2. L'existence éventuelle de plus d'un seul secteur d'utilisation potentiel de l'innovation. Ce sera analysé dans la diffusion potentielle de l'innovation et aura des conséquences, en particulier sur l'intérêt de l'innovation pour une PME, puisque le volume total, éventuellement difficile à gérer pour une PME seule, pourra alors se segmenter en plusieurs volumes accessibles.

système (ainsi, le portable et le téléphone en général sont des innovations systémiques qui nécessitent des centraux, des répartiteurs, des opérateurs, etc.). 
Considérée comme une combinaison de ces trois composantes variables, une rente peut donc être caractérisée par son profil ou configuration (en caricaturant à l'extrême les valeurs prises par les variables): petit ou gros volume; taux de marge élevé ou faible; durée d'exploitation longue ou courte. Ainsi, avec deux possibilités pour chacune des variables, le nombre total de combinaisons est de huit. La recherche entreprise a permis de relever six configurations intéressantes parmi les possibilités de combinaison des variables ${ }^{11}$. Elles sont illustrées dans la figure 3 avec leurs effets de compensation (analyse de la compensation possible) variables entre les composantes volume, taux et durée qui forment la rente.

\section{FIGURE 3}

\section{Typologie des configurations de rente liée aux innovations}

\begin{tabular}{|c|c|c|c|c|c|c|c|}
\hline \multirow{4}{*}{ 1. Avorton } & & - & + & & - & + & \multirow{4}{*}{$\begin{array}{l}\text { 2. Roi du } \\
\text { pétrole }\end{array}$} \\
\hline & volume & & & volume & & & \\
\hline & taux & & & taux & & & \\
\hline & durée & & & durée & & & \\
\hline \multirow{4}{*}{ 3. Gadget } & & - & + & & - & + & \multirow{4}{*}{ 4. Joker } \\
\hline & volume & & & volume & & & \\
\hline & taux & & & $\operatorname{tau} x$ & & & \\
\hline & durée & & & durée & & & \\
\hline \multirow{4}{*}{$\begin{array}{l}\text { 5. Miroir aux } \\
\text { alouettes }\end{array}$} & & - & + & & - & + & \multirow{4}{*}{ 6. Oasis } \\
\hline & volume & & & volume & & & \\
\hline & $\operatorname{tau} x$ & & & taux & & & \\
\hline & durée & & & durée & & & \\
\hline
\end{tabular}

Source: Santi et al., 2003.

11. Les configurations 5 et 6 regroupent des cas où le taux de marge peut être faible ou élevé, parce que les autres variables ont été considérées par tous les acteurs avec lesquels nous avons testé le modèle comme surdéterminantes; en effet, l'effet de compensation volume durée est celui qui semble en premier lieu guider la structuration et le choix de la stratégie mise en place par la PME pour gérer le développement de l'innovation. L'anticipation d'une durée longue permet à la PME de compter sur un flux sûr, même si peu important et lui permet de faire face au risque pérennité que doivent gérer la plupart des PME. Un volume important pendant une durée courte sera envisagé beaucoup plus comme une ressource temporaire, utile pour financer le lancement d'un projet, mais qui devra être relayée très vite. 
Chacune de ces configurations correspond à différents niveaux de volume, taux et durée ainsi qu'à un profil particulier de rente anticipée issue d'une innovation donnée. Comme le montre la figure 3, ces configurations ont été nommées par des appellations imagées permettant aux utilisateurs du modèle de mieux se représenter l'intérêt de la rente considérée ( $c f$. tableau 2).

TABLEAU 2

\section{Les configurations de la rente}

\begin{tabular}{ll}
\hline $\begin{array}{l}\text { Avorton (traduit en anglais } \\
\text { par «schrimp») }\end{array}$ & $\begin{array}{l}\text { Une configuration offrant un petit montant de rente poten- } \\
\text { tielle, avec un volume, un taux et une durée espérés de } \\
\text { faible niveau; l'intérêt de cette configuration est quasiment } \\
\text { inexistant (par exemple, l'Avantime de Renault). }\end{array}$ \\
\hline
\end{tabular}

Roi du pétrole (traduit en anglais par «hampion $»)$

Une configuration avec un fort potentiel de rente (p. ex., la carte à puce); ce profil présente des caractéristiques très séduisantes et pourrait apparaître comme associée à un niveau faible de risque, mais il suppose de pouvoir alimenter une demande anticipée comme importante et peut donc, finalement, se révéler risqué pour une PME seule.

Gadget Une configuration promettant un faible volume et une durée courte, mais un taux de profit élevé; l'intérêt moyen est donc limité et ne justifie pas un fort investissement, en particulier dans une protection, par contre, elle assure temporairement des retours intéressants (exemple cité plus haut: le Hulla hoop). Le taux de profit étant le seul intérêt de ce profil, on portera une attention particulière aux facteurs risquant de l'éroder.

Joker Une configuration avec un volume élevé et une durée longue, mais le faible taux de profit la rend à peine plus attractive que la configuration Gadget (exemple cité plus haut: la poêle Tefal). On veillera ici aux facteurs susceptibles de modifier le volume et la durée.

Miroir aux alouettes (traduit en anglais par «flash in the pan»)
Une configuration avec un bon volume mais une faible durée, le taux étant indifférent (par exemple, les jeux vidéo), constitue un défi pour l'investisseur qui peut être obligé d'engager un capital important pour assurer un retour sur une courte durée; il devra être particulièrement attentif aux facteurs risquant de restreindre le volume.

Oasis

Une configuration présentant une durée importante mais un faible volume et un taux indifférent (exemple cité plus haut: l'appareil Polaroïd). Ses caractéristiques exigent de surveiller l'impact potentiel de facteurs pouvant limiter la durée (technologies concurrentes, par exemple).

Source: Santi et al., 2003. 
L'étude réalisée pour l'INPI suggère que même si la configuration «Roi du pétrole» semble la plus intéressante a priori, la configuration Oasis serait plus adaptée pour une PME en raison du faible volume de vente plus facile à assurer sous contrainte de ressources (Santi et al., 2003). Ce type d'innovation permet en effet à une PME de mieux maîtriser sa croissance et de développer de façon progressive les ressources permettant de financer le succès de son innovation.

Cette étude montre également que la rente peut être notablement érodée lors de l'introduction de l'innovation sur le marché. Il est donc tout à fait essentiel de faire une distinction entre le montant potentiel de rente (estimé a priori, avant introduction sur le marché) et le montant résiduel de rente (après prise en compte des éventuelles résistances apparaissant lors de l'insertion dans l'environnement économique). C'est cette étape d'érosion que nous allons détailler maintenant.

\subsection{2. Érosion de la rente: la rente résiduelle}

La difficulté réelle de nombreux innovateurs réside, d'une part, dans le besoin de convaincre les utilisateurs potentiels d'adopter leur innovation et, d'autre part, dans la confrontation avec les forces du marché, en particulier les pouvoirs de négociations des fournisseurs et des clients (fréquemment sous-évalués, voir Bernasconi et Moreau, 2003). Ces forces concurrentielles caractérisant un marché (Porter, 1980) peuvent être la cause d'une importante érosion de l'avantage concurrentiel anticipé et, donc, de la rente espérée. Le phénomène de diminution de la rente, ou érosion, qui s'en suit, la fait passer d'une rente potentielle à une rente résiduelle.

\section{Les clients}

L'évaluation du pouvoir des clients potentiels suppose l'estimation de la propension générique du marché cible (taux et vitesse d'adoption habituels sur ce marché) à l'adoption des innovations.

Si cette propension est faible, alors, le volume de rente anticipé devra être revu à la baisse. On pourra ainsi étudier ${ }^{12}$ de façon plus attentive le cas d'une innovation type Gadget (intéressante si elle est adoptée vite) ou «miroir aux alouettes» (dont l'intérêt réside dans le volume) confrontées respectivement à un marché adoptant généralement lentement ou peu les nouveautés.

De façon spécifique ensuite, pour un utilisateur potentiel de l'innovation, l'effet de compensation est à évaluer entre ce que l'innovation semble pouvoir lui apporter et ce que son adoption suppose qu'il risque ou abandonne. Le processus de diffusion d'une innovation a été formalisé en cinq étapes par Rogers (1976,

12. Ainsi que suggéré judicieusement par un évaluateur anonyme.

Revue internationale P.M.E., vol. 19, nº 2, 2006 
1995): prise de connaissance, persuasion, décision, mise en œuvre, confirmation. En suivant ces étapes et la façon dont la décision d'adoption se forme dans l'esprit du client, nous avons analysé les caractéristiques de l'innovation qui influencent cette décision.

Nous l'avons synthétisé dans le concept de «valeur d'utilité perçue par le client» (VUPC), calculée par une comparaison entre un «bénéfice perçu» et un «sacrifice perçu» (Ravichandran, 2001). Le bénéfice perçu sera fonction du rapport valeur perçue/prix, de la compréhension de l'innovation par le client (observabilité, testabilité; Ravichandran, 2001; Fichman et Kemerer, 1997), ainsi que de la compatibilité de l'innovation avec les techniques déjà possédées par l'utilisateur (Ravichandran, 2001) ${ }^{13}$.

Si la VUPC résultante est faible, alors le volume de rente sera érodé par l'introduction sur le marché. En effet, les clients ciblés attribueront en fait peu de valeur à l'innovation et l'adopteront peu, en dépit des attentes éventuellement optimistes de l'innovateur.

Un point est en particulier examiné de plus près: c'est la compatibilité de l'innovation avec le système technologique déjà installé dans l'entreprise cliente. En effet, une éventuelle non-compatibilité pourrait être un critère très fort de rejet de l'innovation pour cause de sacrifice perçu très important (Gatignon et Robertson, 1986). Par contre, si l'innovateur réussit à analyser que le risque majeur auquel son innovation devra faire face est une faible VUPC en raison d'une incompréhension technique de l'adopteur potentiel, alors il lui sera possible de travailler tout particulièrement cet aspect. Ravichandran (2001, p. 1) précise ainsi que cette «incertitude technologique perçue par une entreprise peut être modulée par les actions du vendeur de la technologie qui devra éduquer et informer les adopteurs potentiels...».

\section{Les pouvoirs de négociation «portériens»}

Sur un marché caractérisé par de forts pouvoirs de négociation des clients ou des fournisseurs, le taux de profit obtenu grâce à l'innovation sera diminué de manière significative, car ces acteurs seront en mesure de capter le profit pour eux-mêmes (d'imposer un partage de la valeur créée en leur faveur), voire de freiner la diffusion

13. Les bénéfices perçus sont évalués par trois types de critères: l'avantage relatif, la transparence (soit la simplicité à comprendre et à utiliser ainsi que l'essayabilité) et la compatibilité. Les sacrifices perçus sont évalués par trois types de critères: le niveau de prix anticipé, le risque perçu lié à l'adoption (risque de faire le mauvais choix, risque particulièrement fort dans les innovations système - modification des standards, externalités de réseau) et les coûts de transfert (renoncements dans le cas des innovations de substitution). 
de l'innovation. Une meilleure prise de conscience de ce risque peut aider l'innovateur à faire le meilleur choix stratégique: soit nouer une collaboration avec un acteur plus puissant, mais en le choisissant, soit arrêter le développement de l'innovation, soit encore choisir une niche lui permettant d'éviter la confrontation directe avec les acteurs puissants. Une recommandation faite grâce à l'utilisation de l'outil peut être d'ailleurs, en fonction de la rente anticipée, de breveter l'innovation pour avoir une monnaie d'échange avec d'éventuels partenaires (Corbel, 2003).

Une analyse de la menace des substituts potentiels à l'innovation peut se révéler également très utile pour affiner l'estimation de la durée durant laquelle son exploitation pourra donner une rente intéressante. Les processus de réglementation et les éventuelles actions de lobbying caractérisant l'industrie ciblée peuvent aussi avoir un impact sur les trois composantes de la rente potentielle. Les innovateurs ignorent ou sous-estiment fréquemment ces phénomènes qui peuvent pourtant ralentir ou accélérer notablement la diffusion d'une innovation.

De plus, il peut être extrêmement important de considérer le besoin éventuel en complémenteurs (Hax et Wilde II, 2001) en particulier dans le cas d'une innovation systémique. En effet, l'innovation devra alors s'insérer dans un système existant ou être à l'origine d'un nouveau système à créer, pouvant être dominé par un acteur puissant pas forcément prêt à partager la rente.

\subsubsection{Captation de la rente: rente appropriable}

La rente résiduelle subit encore une érosion avant d'être effectivement captée par l'innovateur et devenir la rente appropriable. Cette érosion est le fait des concurrents, d'une part, et de la recherche de ressources, d'autre part.

\section{Le pouvoir des concurrents}

La réaction des concurrents, éventuellement menacés par l'innovation, peut se révéler une difficulté redoutable. Ils peuvent décider de mettre en œuvre des actions de blocage, d'imitation ou de substitutions à l'innovation. C'est d'ailleurs l'un des aspects fréquemment sous-estimés par les innovateurs (voir Bernasconi et Moreau, 2003, p. 26). Selon les analyses de M. Porter et du Boston Consulting Group ${ }^{14}$, l'effet d'érosion sur la rente potentielle dépend de l'intensité concurrentielle régnant dans le secteur ciblé et du type de système concurrentiel auquel appartient le secteur concerné. Il est également essentiel de savoir si l'activité touchée par l'innovation

14. Le Boston Consulting Group a proposé une typologie des systèmes concurrentiels, dite «la $2^{\mathrm{e}}$ matrice du $\mathrm{BCG}$ », dont les critères sont l'existence de barrières à l'entrée, d'une part (pour les systèmes dits «de spécialisation» et «fragmentés») et l'élasticité prix dans le secteur (forte pour les systèmes «de volume» et «de spécialisation»).

Revue internationale P.M.E., vol. 19, nº 2, 2006 
est centrale ou marginale pour les concurrents, car la réaction qu'on peut anticiper de leur part sera alors tout à fait différente et en intensité, et en nature (Rafii et Kampaas, 2002). Là encore, il pourra être recommandé de protéger l'innovation par un brevet, afin de disposer d'un argument pour négocier une coopération ou d'une monnaie d'échange. L'outil présenté ici peut permettre de recommander non seulement le choix de ce type de stratégie, mais aussi l'innovateur à cibler ses interlocuteurs, en fonction des réactions anticipées des concurrents.

Le modèle d'évaluation préconise une estimation précise de la maîtrise par la PME des ressources et compétences nécessaires pour espérer lancer l'innovation avec succès. Cette analyse pourrait en effet révéler un besoin de ressources techniques rares ou l'impératif de chercher une collaboration extérieure pour l'accès à des ressources commerciales ou financières (par exemple, des consultants ou du capital-risque). Il est fréquent que les «dirigeants-propriétaires» de PME préfèrent ne pas avoir recours à une aide extérieure (Mazzarol, 1999; Bougrain et Hauteville; 2002; Katila et Mang, 2003), d'où l'importance de cette étape qui peut les amener à prendre conscience d'un besoin de collaboration dans la recherche de ressources pour lancer une innovation donnée.

La rente ainsi érodée pourra être réellement captée par l'innovateur et pourra seulement alors servir de base à l'évaluation de l'intérêt réel de l'innovation et de la stratégie de valorisation à lui appliquer.

\subsubsection{Recommandations}

Le modèle se conclut donc par une recommandation en matière de valorisation de l'innovation ( $c f$. tableau 3). En fonction du profil de la rente appropriable, il permet de préconiser le choix le mieux adapté parmi les valorisations identifiées: abandon (dans les cas défavorables), valorisation autonome, valorisation partenariale, valorisation déléguée (licences), valorisation cédée (vente de l'éventuel brevet). Les recommandations issues du modèle permettent ainsi de sortir, pour la protection des innovations, de l'alternative «brevet ou pas brevet». En effet, grâce à l'analyse du profil de la rente que pourra vraiment s'approprier l'innovateur et de ses caractéristiques, on peut donner une recommandation bien plus adaptée que le brevet systématique: par exemple, le brevet est fortement recommandé dans le cas où il faut vendre l'innovation, mais plutôt déconseillé dans les cas où la durée de l'innovation est anticipée comme brève.

\subsection{Innovation et PME}

Un point clé dans la recherche que nous avons entreprise est que toutes les innovations ne se valent pas pour une entreprise donnée et, dans le cas particulier d'une $\mathrm{PME}$, toutes les configurations de rente ne sont pas forcément gérables. Le manque

Revue internationale P.M.E., vol. 19, nº 2, 2006 


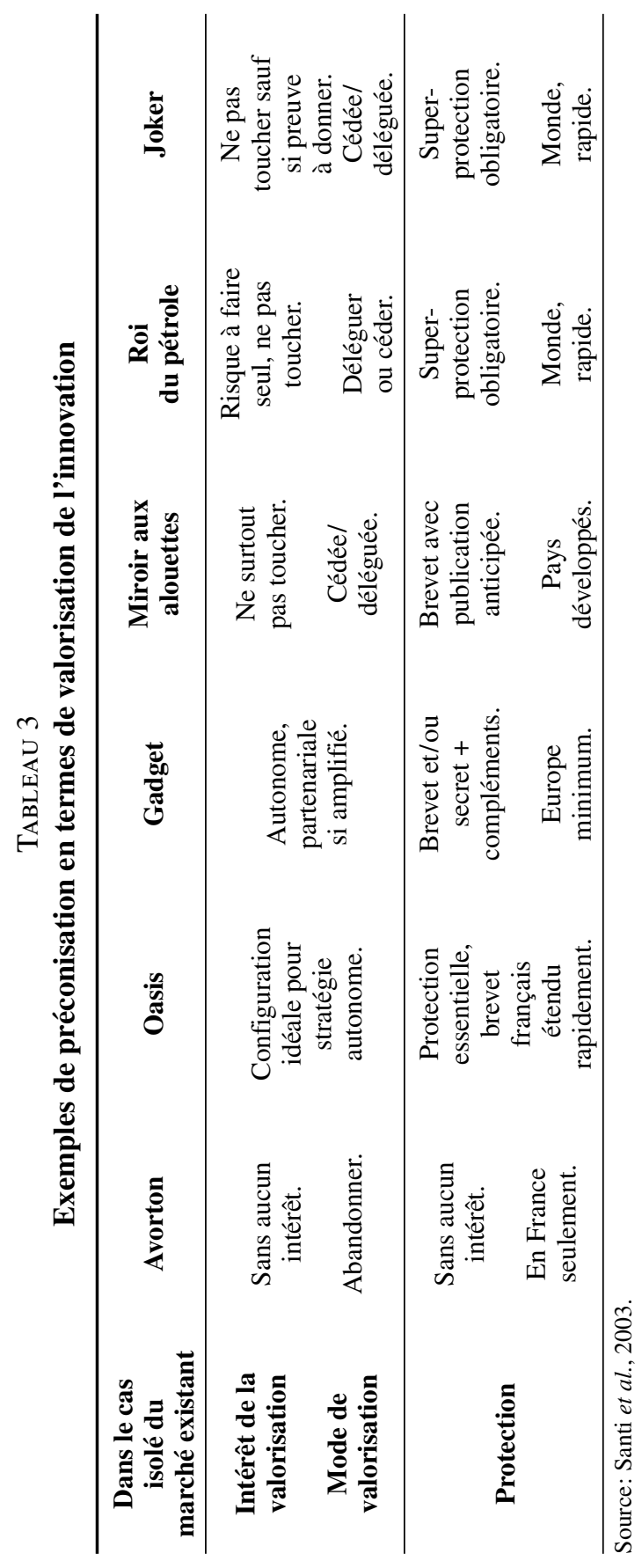

Revue internationale P.M.E., vol. 19, nº 2, 2006 
relatif de ressources disponibles pour une PME moyenne nous a amenés à considérer que la configuration la plus intéressante pour une petite entreprise, qui souvent recherche un développement lent et régulier, est celle intitulée «Oasis» (c'est-à-dire caractérisée par un petit volume, afin d'être en mesure de fournir les ressources nécessaires et une durée d'exploitation longue). Toutes les autres configurations présentent un ou plusieurs dangers potentiels pour une PME. Par exemple, la configuration Avorton est inintéressante pour la plupart des entreprises et la configuration Gadget n'offre qu'une durée de vie courte, ce qui incite à recommander une stratégie d'écrémage du type «take the money and run». Les trois autres configurations - Miroir aux alouettes, Joker et Roi du pétrole - sont toutes caractérisées par un gros volume potentiel souvent difficile à assurer par de petites entreprises ayant des capacités de production et des moyens commerciaux limités. En revanche, elles peuvent prendre tout leur intérêt dans le cadre d'une coopération ou pour une entreprise cherchant un développement rapide. Dans ces deux cas, les recommandations issues de l'outil de diagnostic devraient permettre à la PME de choisir la meilleure stratégie de valorisation de son innovation tout en sachant parfaitement à quoi s'attendre. Le modèle construit permet ainsi d'intégrer la réflexion en matière de protection et de valorisation de l'innovation à la stratégie de l'entreprise puisqu'en fonction de l'érosion de la rente potentielle anticipée lors de l'évaluation d'une innovation, il donne des recommandations stratégiques différenciées et adaptées.

\subsection{Validation du modèle (par des experts, puis par des managers)}

Au cours de sa conception et pour en affiner la pertinence, cet outil d'évaluation et de recommandation a été dans un premier temps testé auprès d'un panel d'experts en propriété industrielle. Nous avons rencontré une vingtaine d'entre eux, qui se sont déclarés fort intéressés, dont l'avis a permis de faire évoluer le modèle marginalement ( $c f$. annexe I) puis, dans un deuxième temps, il a été appliqué à un échantillon de 15 dirigeants de PME françaises au printemps 2002 ( $c f$. annexe II), qui ont reconnu l'intérêt de la logique générale de la démarche et ont, par leurs recommandations, permis d'affiner certaines phases. Dans le cas de ces PME, l'outil a été utilisé aussi bien ex ante qu'ex post, afin de vérifier le pouvoir de prédiction de l'outil. Cette utilisation a permis à plusieurs managers de comprendre a posteriori des réactions concurrentielles qu'ils n'avaient pas anticipées, mais dont l'outil montrait qu'elles étaient difficilement évitables ${ }^{15}$.

15. Dans un cas, essentiellement parce que l'innovation considérée attaquait de front un concurrent sur une activité vitale pour lui et qu'il avait des moyens et ressources de nature et quantité suffisante pour réagir fortement. Dans un autre cas, le directeur de la PME innovante n' avait pas réalisé qu'en développant son innovation, il concurrençait directement ses propres clients, s'exposant ainsi à une réaction facile à anticiper. 
Au total, l'étude menée a montré que cette démarche d'analyse était une bonne façon d'évaluer en amont le risque encouru par un innovateur et que la précision des estimations était considérée comme suffisante pour un outil d'aide précoce à la décision. Cependant, la question reste de savoir si les dirigeants de PME évaluent en temps normal le risque qu'ils courent en lançant une innovation et comment. Autrement dit, lorsqu'ils ne disposent pas de cet outil, les managers de PME innovateurs prennent-ils des risques inconsidérés? Font-ils des choix judicieux? Peut-on caractériser le management de l'innovation de ceux qui semblent faire les «bons» choix?

\section{Suites de la recherche}

C'est pour approfondir cette idée que nous avons choisi d'utiliser ce modèle pour analyser le management de l'innovation dans des PME. Dans une recherche lancée au printemps 2003, en Australie occidentale, dont l'économie est très liée à la vitalité des PME, nous avons choisi de construire un questionnaire plus quantitatif, basé sur le modèle réalisé pour l'INPI et le complétant avec des questions sur le mode de management de l'innovation des PME interrogées.

\subsection{Conception d'une étude quantitative}

Nous avons donc construit un questionnaire détaillant chacune des étapes du modèle pour une innovation particulière de la PME interviewée ( $c f$. annexe III). En complément de l'évaluation du risque, le questionnaire comporte des questions portant sur le contexte de décision et le processus de décision du dirigeant de PME. Les questions s'appuient sur les concepts et résultats issus des travaux de Ajzen et Fishbein (1980) dont la Théorie de l'action raisonnée (Theory of Reasoned Action ou TRA). Cette partie de l'étude vise à déterminer les influences relatives des Attitudes (par exemple, croyance et évaluations rationnelles) et des Normes subjectives (par exemple, croyances normatives et motivation pour se conformer à des influences externes). Des recherches précédentes ont montré que les dirigeants de PME sont susceptibles d'être plus influencés par des Attitudes que par des Normes subjectives, ce qui pourrait indiquer que les influences extérieures pourraient être plus faibles que les convictions de l'entrepreneur et son propre jugement (Thompson et Panayiotopoulos, 1999). Néanmoins, l'incertitude caractérisant la plupart des innovations pourrait suggérer que le fait d'avoir faiblement recours à des avis de conseillers extérieurs risque d'être dommageable pour l'entreprise.

Ce questionnaire comprend donc cinq parties (voir annexe III):

- quatre sur les étapes du modèle: la première décrit l'entreprise, la deuxième, l'innovation (évaluant la rente potentielle), la troisième analyse l'érosion 
de la rente par les forces du marché (hors concurrence, évaluant la rente résiduelle) et la quatrième, l'érosion de la rente par la concurrence et le manque de ressources de la PME (évaluant la rente appropriable);

- une sur le mode de décisions et de management de l'innovation dans la PME.

Le modèle utilisé étant basé sur des anticipations qualitatives et approximatives, les questions en suivent très fidèlement la logique et sont à échelle de Likert de trois à sept niveaux (le nombre de niveaux variant selon le degré de précision possible de l'anticipation) complétées des questions binaires de synthèse.

Il faut néanmoins souligner un certain nombre de limites, certaines liées à l'outil lui-même, d'autre, à l'étude. D'abord, la complexité de l'outil d'analyse et son objectif premier font qu'il est conçu pour être utilisé avec le soutien d'un conseiller, ce qui en limite l'utilisation et pose quelques questions sur la capacité des $\mathrm{PME}$ à répondre correctement aux questions posées. Ensuite, il s'appuie essentiellement sur des évaluations ex ante et qualitatives. Il suppose donc une très grande connaissance du secteur et des acteurs concernés; le plus souvent, les dirigeants de PME connaissent ces informations, mais cela pourrait ponctuellement ne pas être le cas. Le passage de l'outil de conseil au questionnaire pose lui aussi quelques problèmes de perte d'information, ou d'acuité des critères d'analyse choisis.

Pour vérifier que cette limite n'était pas susceptible de retirer tout intérêt à l'étude quantitative, nous avons dans un premier temps mené une enquête pilote auprès de quelques PME innovantes d'Australie occidentale, complétée par des entretiens avec des entrepreneurs locaux impliqués dans l'innovation technologique. L'objectif de cette partie de l'étude a été de vérifier que l'outil tel qu'il a été développé en France était adapté à l'environnement économique ouest-australien et compréhensible sans l'aide d'un expert pour présenter les questions. Les résultats de cette enquête-pilote suggèrent que l'outil d'évaluation possède un très bon potentiel d'application et une bonne utilité pour aider les petites entreprises à évaluer les mérites relatifs d'un projet d'investissement dans une innovation. Il est intéressant de noter, par exemple, que l'un des entrepreneurs rencontrés a demandé à l'équipe de chercheurs l'autorisation d'utiliser le modèle d'analyse et ses résultats dans une négociation avec des partenaires potentiels, trouvant les arguments utilisés particulièrement convaincants ${ }^{16}$. Par ailleurs, les résultats semblent confirmer que

16. Les remarques présentées par cet entrepreneur étaient à rapprocher de façon très directe de l'argument développé par Guilhon et Montchaud (2003) et par Bach et Lambert (2000) sur la difficulté à réduire l'asymétrie d'information entre le partenaire financeur potentiel et l'innovateur. L'outil d'analyse du risque proposé semblait, selon cet entrepreneur, susceptible de réduire de façon crédible cette asymétrie d'information ex ante ou, selon le concept de Lerner (1998), d'«information gap». 
les dirigeants de PME, lorsqu'ils prennent des décisions stratégiques, ne se fient à des influences externes que pour soutenir et renforcer leurs décisions intuitives en matière d'investissement dans une innovation. Cela nous a semblé confirmer l'intérêt qu'il pouvait y avoir à croiser, d'une part, l'analyse des innovations produites par les PME et, d' autre part, l'analyse de leur mode de management de l'innovation. Nous avons donc poursuivi l'étude par l'envoi du questionnaire ${ }^{17}$.

Durant la fin de l'année 2003, le questionnaire a été envoyé à environ 500 PME reconnues comme innovantes. Ces entreprises ont été choisies avec l'aide du ministère de l'Industrie et des Ressources d'Australie occidentale (WA State Government Department of Industry and Resources), qui a grandement aidé à la collecte des données. L'étude, démarrée à la fin du mois d'août, est encore en cours mais le taux de retour avait dépassé les $12 \%$ dès la mi-novembre. Le premier envoi par courrier a été complété par un courrier électronique et une mise en ligne du questionnaire par l'intermédiaire du Ministère.

\subsection{Conclusion et résultats attendus}

Grâce à ce questionnaire, nous espérons caractériser, d'une part, l'activité d'innovation elle-même des PME répondantes et donc, chercher à voir quels types d'innovation elles choisissent de développer et, d'autre part, croiser cette caractérisation avec le mode de décision et de management de l'innovation qu'elles pratiquent. Par exemple, est-ce que les PME lancent plutôt des innovations de type «oasis»? Est-ce que les PME qui lancent des innovations «oasis» ont un mode de management de l'innovation différent des autres? Peut-être plus structuré ou plus formalisé? À qui demandent-elles conseil le plus souvent en matière d'innovation? Cela a-t-il une conséquence sur le type d'innovation qu'elles développent?

Ces différents éléments sont attendus avec impatience à la fois par les managers eux-mêmes, mais aussi par différents réseaux de conseil et soutien à l'innovation des PME australiens. Ces derniers attendent des éléments permettant d'aider les PME à orienter leurs efforts en matière d'innovation et de pratique technique, ce qui permet d'espérer des progrès en termes de compétitivité et de pertinence des choix stratégiques (Lagacé et Bourgault, 2003).

17. Cette enquête a été menée durant les mois de juillet et août 2003 grâce à des entretiens avec des PME ouest-australiennes contactées avec l'aide du ministère de l'Industrie (Department of Industry and Resources of Western Australia). 


\section{Annexes}

\section{AnNeXe I \\ Les entretiens «expert»}

\section{Les 20 entretiens réalisés auprès d'un échantillon diversifié d'experts} de novembre 2002 à janvier 2003.

$\rightarrow 8$ : cabinets de conseil PI

$\rightarrow 5$ : grandes entreprises (PI)

$\rightarrow 2$ : capital-risque et essaimage

$\rightarrow 3$ : incubation et valorisation

$\rightarrow 2$ : académique - institutionnel

\section{Objectif des entretiens}

1. Enrichir notre modèle en recueillant et rassemblant les approches respectives de valorisation d'une innovation de la part de ces experts.

2. Tester notre démarche méthodologique et recueillir leurs remarques et leurs critiques par rapport à notre approche et aux concepts utilisés.

3. Enrichir notre connaissance et notre approche sur les mécanismes de protection intellectuelle et valider la part PI de nos stratégies de valorisation.

\section{L'entretien}

Un déroulement en trois temps:

Partie ouverte

Critères d'évaluation, méthodologie des experts.

Test du modèle (partie structurée)

Présentation de la structure et des concepts de base du modèle, dialogue, échanges, test d'idées nouvelles et prise de note des remarques.

Et la PI dans tout cela (synthèse ouverte)?

Comment la PI s'insérerait-elle le mieux dans notre démarche?

\section{Résultats}

En fonction des recommandations des experts consultés, la modélisation du raisonnement des innovateurs a été affinée. En particulier, la recommandation principale a été d'aller vers une simplification de l'outil. 


\section{ANNEXe II \\ Les entretiens «PEI»}

1. Entretiens réalisés auprès d'un échantillon de 15 dirigeants de PEI:

- Électronique et informatique

- Biens de consommation courante

- Équipements municipaux

- Biotechnologie

- Services

\section{Un déroulement en deux temps}

1. Présentations

- Présentation de la recherche pour l'INPI

- Présentation de l'entreprise

2. Test du modèle

- Déroulement du modèle

- Discussion des réactions

- Prise de notes

\section{Synthèse de l'analyse des résultats}

Forces

- Les recommandations sont pertinentes.

- Le tableau de caractérisation (en termes de rente) des innovations est éclairant et reste clé pour le modèle.

- Les matrices sont jugées claires et compréhensibles.

- Les logigrammes (d'évaluation des ressources de la PEI) sont particulièrement appréciés.

Faiblesses

- Il faut l'expert à côté du modèle pour en traduire les termes et la logique.

- Le modèle supporte bien l'analyse, mais il est parfois faible sur les recommandations.

- Les termes ne sont pas assez parlants et on n'a pas d'exemples pour les expliquer.

- La question de la segmentation n'est pas suffisamment clairement abordée. 


\section{ANNEXE III \\ Brève description du questionnaire adressé aux PME australiennes}

\section{A. «The nature of your firm»}

Six questions de démographie fermées avec liste ou ouvertes

Exemple

Q4: Has your firm introduced or engaged in any of the following within the past three years?
A. The preparation of a formal business plan.
YES $\square 1 \quad N O \square 2$
$B$. Exporting or engagement in an international market.
YES $\square 1 \quad N O \square 2$
C. Training of yourself in a formal management development program.
YES $\square 1 \quad$ NO $\square 2$
D. Training of key employees in a formal management development program.
YES $\square 1 \quad N O \square 2$
E. Joint ventures or formal partnerships with leading customers.
YES $\square 1 \quad N O \square 2$
F. Joint ventures or formal partnerships with key suppliers.
YES $\square 1 \quad$ NO $\square 2$
G. Joint ventures or formal partnerships with other companies.
YES $\square 1 \quad$ NO $\square 2$
$H$. Raising of venture capital requiring equity sharing with other parties.
YES $\square 1 \quad N O \square 2$

\section{B. «The nature of innovation within your firm»}

Deux questions binaires sur le type et la fréquence des innovations depuis trois ans dans la PME.

Q7: Has your firm introduced any of the following innovations within the past three years, and what have been the frequency and the level of investment in such innovations? (Please indicate for as many innovations as applicable.)

$\begin{array}{cc}\text { Number of } & \text { \% of annual } \\ \text { innovations over } & \begin{array}{l}\text { turnover } \\ \text { invested } \\ \text { past three years }\end{array} \\ \text { peryear }\end{array}$

A. Product-service innovations - e.g. new products developed from research or introduced to market.

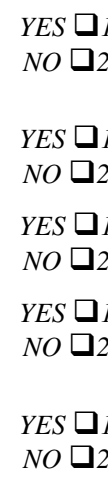

YES $\square 1$

$\%$

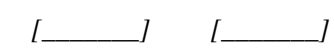

B. Market development innovations - e.g. entering new markets with existing products, exporting.

YES $\square 1$ NO $\square 2$

C. Marketing innovations - e.g. development of a new brand or use of databases for marketing.

YES $\square 1$

NO $\square 2$

YES $\square 1$

NO $\square 2$ tion of new tools or methods, use of computer controlled manufacture.

YES $\square 1$

NO $\square 2$

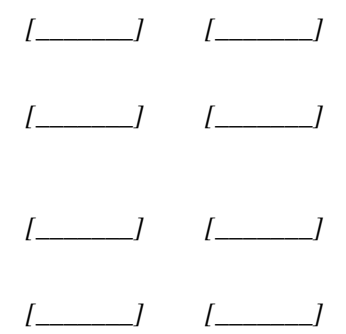

F. Other

Revue internationale P.M.E., vol. 19, nº 2, 2006 
(Les questions suivantes concernent une innovation en particulier.)

Trois questions binaires sur sa nature.

Cinq questions avec échelle de Likert à cinq niveaux sur le volume potentiel.

With respect to the potential volume of sales for this innovation (as refered to in Q8):

Q12: In your estimation what would be the potential market adoption rate of this innovation within your sector?

Very limited $\square 1$

Limited $\square 2$

Medium $\square 3$

Wide $\square 4$

Very wide $\square 5$

Une question avec échelle de Likert à cinq niveaux sur le taux.

Deux questions avec échelle de Likert à cinq niveaux sur la durée.

\section{C. «Bringing the innovation to market»}

Trois questions avec échelle de Likert à trois niveaux sur les clients potentiels, quelle que soit l'innovation.

Q21: When these firms do adopt, the proportion of adopting firms is usually ...

Sept questions avec échelle de Likert à sept niveaux sur la réaction anticipée des clients potentiels quant aux bénéfices qu'ils pensent retirer de cette innovation.

Une question binaire de synthèse sur l'espoir de bénéfices.

Huit questions avec échelle de Likert à sept niveaux sur la réaction anticipée des clients potentiels quant aux sacrifices qu'ils pensent devoir faire en adoptant cette innovation.

Q28: Customers will view your proposed innovation's compatibility with existing technologies as being...

Extremely

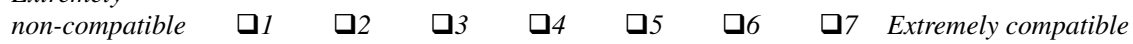

Une question binaire de synthèse sur les sacrifices potentiels

Seize questions avec échelle de Likert à sept niveaux sur les rapports de force respectifs du secteur (six sur l'influence sur la qualité, trois sur les coûts de transfert, trois sur la menace d'intégration, trois sur l'importance du CA espéré).

Q39: The influence that key suppliers have over cost control in this industry is...

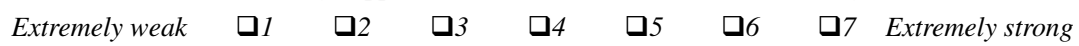

Une question binaire de synthèse sur le rapport de force

Trois questions binaires sur la menace de substituts.

Trois questions binaires et une à sept niveaux sur le pouvoir des complémenteurs.

Cinq questions binaires sur l'influence de la réglementation.

\section{D. «Competitors»}

Six questions à trois choix sur la valeur (l'intérêt) de l'activité.

Quatre questions binaires sur les principaux concurrents.

Revue internationale P.M.E., vol. 19, nº 2, 2006 
Q68: With respect to your competitors business portfolios, the proposed innovation you are considering would be ...

A core business $\square 1$ A marginal business $\square 2$

Dix questions binaires sur les ressources et compétences de la PME (et sa capacité à développer seule l'innovation).

\section{E. «The nature of strategic decision-making in your firm»}

Treize questions avec échelle de Likert à sept niveaux sur le processus de décision interne quant à cette innovation dans la PME.

Q82: How much power do you personally have within your firm over whether this innovation will be undertaken?

$\begin{array}{lllllllll}\text { Not very much power } & \square 1 & \square 2 & \square 3 & a_{4} & \square 5 & \square 6 & \square 7 & \text { A great deal of power }\end{array}$

Quatorze questions binaires sur l'influence des acteurs externes à l'entreprise quant à cette innovation.

\section{Bibliographie}

AjZen I. et M. FishBeIN (1980), Understanding Attitudes and Predicting Behaviour, Englewood Cliffs, N.J., Prentice Hall, 278 p.

APEC (2003), Small Business and Trade in APEC: A Report Highlighting the Contribution of Medium, Small and Micro Enterprises to the Asia Pacific Region, Singapore, APEC.

ARTHUR, W.B. (1996), «Increasing returns and the new world of business », Harvard Business Review, vol. 74, no 4, juillet-août, p. 100-109.

BACH, L. et G. LAMBERT (2000), «Étude transversale des facteurs de l'innovation: forces et faiblesses respectives des PME et des grandes entreprises», dans Les PME face à l'innovation et à la gestion des connaissances: quel rôle jouent les grandes structures?, Actes du XII ${ }^{\text {e }}$ Séminaire annuel OI2, Université technologique de Compiègne, 24-27 janvier.

Beaudoin, R. et J. St-Pierre (1999), «Financement de l'innovation dans les PME, une recension récente de la littérature», Trois-Rivières, Rapport de veille présenté à l'Observatoire économique Canada, Développement économique Canada.

Bernasconi, M. et F. Moreau (2003), «L'évolution du projet des jeunes entreprises technologiques innovantes au cours des premières années: une méthode d'appréciation du cheminement stratégique», Revue internationale PME, vol. 16, $\mathrm{n}^{\text {os }} 3$-4, p. 11-29.

Bougrain, F et B. Hauteville (2002), «Innovation, collaboration and SMEs internal research capacities», Research Policy, n 31, p. 735-747.

Corbel, P. (2003), «Le brevet: un outil de coopération/exclusion», présenté au Colloque «Les stratégies concurrentielles: nouvelles approches, nouveaux enjeux», IRG/PRISM, Marne-la-Vallée, novembre 2003.

DAGNiNO, G.B. (1996), «Understanding the economics of Ricardian Chamberlinian and Schumpeterian rents: implications for strategic management», Rivista Internazionale di Scienze Economiche e Commerciali, vol. 43, n 1, p. 213-35.

Revue internationale P.M.E., vol. 19, nº 2, 2006 
DruCKer, P.F. (2002), «They're not employees, they're people», Harvard Business Review, vol. $80, \mathrm{n}^{\circ} 2$, p. 70-77.

DziUra, M.J. (2001), «Innovations: sources and strategies», International Journal of Technology Management, vol. 21, $\mathrm{n}^{\mathrm{os}}$ 5-6, p. 612-628.

EUROPEAN COMMISSION (2004), «Innobarometer 2004», Flash Eurobarometer 164 - TNS Sofres/EOS Gallup Europe, novembre.

Fichman, R.G. et C.F. Kemerer (1997), «The assimilation of software process innovations: an organizational learning perspective», Management Science, vol. 43, $\mathrm{n}^{\circ} 10$, p. $1345-1363$.

FreEL, M.S. (2000), «Do small innovating firms outperform non-innovators?», Small Business Economics, vol. 14, nº 3, p. 195-210.

Gatignon, H. et T.S. RoberTson (1986), «Competitive effects on technology diffusion», Journal of Marketing, vol. 50, $\mathrm{n}^{\circ}$ 3, juillet, p. 1-12.

Gatignon, H. et T.S. RoberTson (1993), «The impact of risk and competition on choice of innovations», Marketing Letters, vol. 4, no 3, p. 191-205.

Guilhon, B. et S. Montchaud (2003), «Le capital à risque et les jeunes entreprises innovantes: problématiques et enjeux», Revue internationale PME, vol. 16, ${ }^{\text {os }} 3-4$, p. 53-73.

Hansen, O.E., B. Sondergard et S. Meredith (2002), «Environmental innovations in small and medium sized enterprises», Technology Analysis and Strategic Management, vol. $14, \mathrm{n}^{\mathrm{o}} 1, \mathrm{p} .37-56$.

HAX, A. et D. WILDE II (2001), «The Delta model - discovering new sources of profitability in a networked economy», European Management Journal, vol. 19, n 4, p. 379-391.

Henderson, R.M. et K.B. ClarK (1990), «Architectural innovation: the reconfiguration of existing product technologies and the failure of established firms», Administrative Science Quarterly, vol. 35, no 1, mars, p. 9-30.

HuET, F. (2004), «Innovation through cooperation in SMEs: the central role of entrepreneurial dynamism», Actes du Séminaire OI2, Costech, Université technologique de Compiègne.

JENSEN, R. (1982), «Adoption and diffusion of an innovation of uncertain profitability», Journal of Economic Theory, vol. 27, n 1, p. 182-193.

Kahneman, D. et D. Lovallo (1993), «Timid choice and bold forecasts: a cognitive perspective on risk taking», Management Science, vol. 39, $\mathrm{n}^{\circ}$ 1, p. 17-31.

Katila, R. et P. Mang (2003), «Exploiting technological opportunities: the timing of collaborations», Research Policy, $\mathrm{n}^{\circ}$ 32, p. 317-332.

KLinke, A et O. RenN (2002), «A new approach to risk evaluation and management: riskbased, precaution-based, and discourse based strategies», Risk Analysis, vol. 22, $\mathrm{n}^{\circ} 6$, p. 1071-1094.

LAGACÉ, D. et M. Bourgault (2003), «Linking manufacturing improvement programs to the competitive priorities of Canadian SMEs», Technovation, vol. 23, no 8, p. 705-715.

LERNER, J. (1998), «Angel financing and public policy: an overview», Journal of Banking and Finance, vol. 22, p. 773-783. 
LewIN, P. et S.E. Phelan (2002), «Rent and resources: an Austrian perspective», University of Texas at Dallas, octobre, <http://www.utdallas.edu/ plewin/AustFirm>.

Martin, S. et J.T. ScOTT (2000), «The nature of innovation market failure and the design of public support for private innovation», Research Policy, $n^{\circ} 29$, p. 437-447.

Mazzarol, T.W. (1999), «Partnerships: a key to growth in small business», Journal of Enterprising Culture, vol. 7, $\mathrm{n}^{\circ}$ 2, p. 105-125.

Miles, M.P., C.W. Paul et A. Wilhite (2003), «Modelling corporate entrepreneurship as rent-seeking competition», Technovation, vol. 23, n 5, p. 393-400.

MockLER, R.J. (2003), «Prescription for disaster: failure to balance structured and unstructured thinking», Business Strategy Review, vol. 14, n 2, p. 17-26.

MogeE, M.E. (2000), «Foreign patenting behaviour of small and large firms», International Journal of Technology Management, vol. 19, $\mathrm{n}^{\text {os }}$ 1-2, p. 149-164.

NCOE (2000), Embracing Innovation: Entrepreneurship and American Economic Growth, Washington D.C., National Commission on Entrepreneurship White Paper.

Nemeth, C.J. (1997), «Managing innovation: when less is more», Californian Management Review, vol. 40, n 1 .

PAvitT, K. (1984), «Sectoral patterns of technical change», Research Policy, no 13, p. 343-373.

Porter, M.E. (1980), Competitive Strategy: Techniques for Analyzing Industries and Competitors, New York, The Free Press, 396 p.

RAFII, F. et P.J. KAMPAS (2002), «How to identify your enemies - before they destroy you», Harvard Business Review, novembre.

RAVICHANDRAN, T. (2001), «Innovation assimilation in the presence of knowledge barriers, technology uncertainty and adoption risks», Academy of Management Proceedings.

Rogers, E.M. (1976), «New product adoption and diffusion», Journal of Consumer Research, vol. 2, n ${ }^{\circ}$, p. 290-301.

Rogers, E.M. (1995), Diffusion of Innovations, $4^{\mathrm{e}}$ edition, New York, The Free Press.

Santi, M., S. Reboud, H. Gasiglia et A. SAbouret, (2003), «Modèle de valorisation et de protection intellectuelle des innovations des PEI», HEC/INPI, juillet, 63 p. Site Web: <http://www.ccip.fr/ccmp/detail.asp?id=G1204> et <http://www.hec. $\mathrm{ca} /$ provaluor $>$.

Schumpeter, J.A. (1912), Theorie der Wirtschaftlichen Entwicklung, Leipzig, Dunckler und Humblot; traduit en anglais, The Theory of Economic Development, New York, Oxford University Press, 1961.

Shapiro, C. et H. VARIAn (1998), A Strategic Guide to the Network Economy, Boston, Mass., Harvard Business School Press.

Thompson, K.E. et P. Panayiotopoulos (1999), «Predicting behavioural intention in a small business context», Journal of Marketing Practice: Applied Marketing Science, vol. $5, \mathrm{n}^{\circ} 3$, p. 89-96.

Tornatzky, L.G. et M. Fleischer (dir.) (1990), The Processes of Technological Innovation, Toronto, Lexington Books, 298 p.

Revue internationale P.M.E., vol. 19, nº 2, 2006 